\title{
Effet des facteurs physiques de l'environnement sur les premiers stades de la régénération naturelle du sapin pectiné (Abies alba Mill) dans certaines stations du Jura
}

\author{
BS Tan, S Bruckert \\ Université de Franche-Comté, laboratoire de pédologie, place Leclerc, \\ 25030 Besançon cedex, France
}

(Reçu le 9 juillet 1991; accepté le 10 décembre 1991)

\begin{abstract}
Résumé - Les premières phases de la régénération du sapin ont été analysées en étudiant la germination et le comportement des semis dans des expériences menées à la fois au champ en environnement naturel et au laboratoire en conditions contrôlées. Pour atteindre ces objectifs, le vaste domaine karstique du Jura a constitué un milieu modèle de choix parce que des stations forestières typiques sont observées côte-à-côte, avec des niveaux de régénération, soit très élevés, soit très bas. L'absence de géotropisme à la germination détermine à la fois la morphologie «en crosse» observée sur les semis naturels et le comportement ultérieur des semis. Les radicules qui rampent sur le substrat sans s'y implanter, rendent les semis très sensibles aux agents physiques, parmi lesquels les orages et les ensoleillements printaniers sont les plus efficaces pour entraîner des lésions et la dessiccation des semis. Le bilan de la régénération apparaît significativement modulé par l'humidité relative et la température de l'air, l'ensoleillement pendant le mois qui suit la germination, puis par le pédoclimat pendant les mois chauds qui agissent sur les semis mal enracinés. In vitro, il est démontré que l'humidité de l'air constitue le facteur qui détermine la survie des semis (premiers stades de développement) et que l'humidité du sol constitue le facteur qui détermine le développement racinaire et la résistance des plantules à des stress hydriques.
\end{abstract}

régénération naturelle / Abies alba / enracinement des semis / stress hydrique / humidité de l'air / pédoclimat / Jura

Summary - Effect of physical environmental factors on the initial stages of the natural regeneration of fir (Abies alba) at some sites in the Jura. The early stages of fir regeneration were analysed in order to study the germination and the seedling behaviour in the field and in the greenhouse. The large karstic area of the Jura offers typical forest sites with high regeneration levels adjacent to those with low regeneration levels (fig 1). The lack of geotropism which is observed on seed germination induces the "grip" morphology of natural seedlings and their subsequent behaviour. The primary root extends over the substrate without penetrating it, and so appears to be susceptible to physical factors such as rain storms and insolation which damage and dry out the seedlings (tables $I V$ and $V$ ). The regeneration balance was found to be significantly influenced by air humidity, air temperature and insolation during the first month following germination and later by the soil environ- 
ment during the hot periods which could result in elimination of superficially rooted seedlings (fig 2). Laboratory measurements (fig 3) showed that air humidity was the determinant factor in seedling survival and soil humidity the parameter influencing root growth and resistance to moisture stress.

natural regeneration / Abies alba / seedling rooting / moisture stress / air humidity / soil environment/Jura

\section{INTRODUCTION}

Les difficultés de régénération du sapin pectiné (Abies alba Mill) qui affectent l'ensemble des massifs européens de moyenne montagne, ont fait l'objet de nombreuses recherches. Les facteurs impliqués dans le phénomène seraient physiques (Gabellini et Screm, 1968; Ferrari et Wolf, 1970; Tan, 1987), chimiques (Rousseau, 1960; Vallée, 1967) et/ou biotiques, d'origine végétale (Brinar, 1971; Becker et Bennett, 1980; Becker et Drapier, 1984, 1985) ou animale (Badre, 1962; Wojnar, 1970; Tan, 1987; Roques, 1988). Les chercheurs sont d'accord pour penser que ce sont des interactions entre diverses causes possibles qui déterminent le niveau de régénération. Néanmoins, les facteurs interviennent dans le milieu naturel de façon échelonnée dans le temps, et ceux qui agissent en premier sont déterminants. De là découle l'intérêt d'étudier les processus qui se déroulent pendant les premières phases de la régénération. Cet objectif a été visé dans le contexte des plateaux calcaires jurassiens parce qu'on y rencontre des modèles géomorphologiques et stationnels qui influencent le bilan de la régénération (Tan, 1988). Les plateaux se composent, à parts égales, de surfaces tabulaires subhorizontales (position topographique sommitale de plateau proprement dit) et de dépressions karstiques (combes, ensemble de dolines), réparties régulièrement en alternance (Bruckert et Gaiffe, 1980). En position sommitale, la régénération semble dépendante de l'environnement pédologique, lui- même inféodé aux faciès des roches calcaires (Gaiffe et Bruckert, 1991). En position de dépression, les sols profonds et bien drainés sont, selon les stations, boisés ou non. Certaines combes sont impropres à l'état boisé : elles accumulent l'air froid et subissent de sévères gelées tardives, jusqu'à $-10^{\circ} \mathrm{C}$, en été (Tan, 1983). En dehors de ces cas, la plupart des dépressions karstiques ont été ou sont boisées, les sols se caractérisant par des potentialités forestières élevées, mais la régénération naturelle du sapin s'y produit de façon très inégale (Tan, 1988). On y rencontre notamment deux stations dont les niveaux de régénération apparaissent diamétralement opposés : ce sont les "fonds de dolines" caractérisés par leur inaptitude à la régénération et les «replats» remarqués au contraire pour leur aptitude à porter de jeunes sapins vigoureux. Dans de tels sites, dont les sols de type colluvial à mull mésotrophe sont identiques génétiquement et physico-chimiquement, il était prévisible que d'autres paramètres, comme le microclimat et le pédoclimat, pouvaient contrôler la régénération.

C'est pourquoi, nos objectifs ont été de suivre in situ la germination des graines et le comportement des semis dans l'environnement des stations étudiées, de mesurer in situ les paramètres climatiques stationnels des sites favorables ou non à la régénération, et d'analyser in vitro l'action des paramètres climatiques et pédoclimatiques mis en cause et leurs effets sur le développement des plantules de sapin, par une expérimentation en conditions contrôlées. 


\section{MATÉRIEL ET MÉTHODES D'ÉTUDE}

\section{Caractéristiques des stations}

Les sites expérimentaux ont été sélectionnés à partir de l'étude de 128 stations (Tan, 1988) réparties dans la forêt domaniale de la Joux qui occupe $135 \mathrm{~km}^{2}$ sur le plateau de Levier à une altitude d'environ $800 \mathrm{~m}$. Cette forêt de la Joux, une hêtraie-sapinière, comprend des futaies ouvertes de 100 ans et de 120-140 ans, la strate arborescente couvrant $70 \%$ de la surface au sol. Les tiges se composent de sapins (80\%), d'épicéas $(14 \%)$ et de feuillus $(6 \%)$. Les arbres occupent le terrain en laissant de larges vides dans les dépressions karstiques. Phytosociologiquement, la forêt se rattache à l'Abieti-Fagetum elymetosum avec des variantes fraîches, dans les dépressions, à Primula elatior, Stellaria nemorum, Veronica montana, Circea lutetiana.

Le climat est de type tempéré très humide et froid, avec $1700 \mathrm{~mm}$ de précipitations moyennes annuelles et $6^{\circ} \mathrm{C}$ de température moyenne annuelle. L'évapotranspiration potentielle, selon Thornwaithe, étant de $579 \mathrm{~mm}$, le drainage climatique annuel est élevé. Cependant, en été et en automne, la probabilité d'avoir 3 séquences de 3 jours secs, ie avec moins de $1 \mathrm{~mm}$ de pluie par jour, dépasse $66 \%$ d'après la Météorologie nationale de Besançon.

Le substratum géologique est constitué essentiellement par les calcaires blancs à pâte fine du Bathonien (Jurassique moyen), caractéri- sés par leur aptitude à l'altération pelliculaire (Bruckert et Gaiffe, 1980) et la karstification (Aubert, 1969; Nicod, 1990).

Les expériences portent plus particulièrement sur 2 types de stations qui appartiennent aux dépressions karstiques et qui s'opposent par leur végétation et leur niveau de régénération naturelle du sapin (fig 1). Les stations de "fond de doline" forment des creux concaves à l'intérieur des dépressions karstiques. Leur sol est colonisé par une végétation dite cde hautes herbes" en été (Athyrium filix femina, Circea lutetiana, Senecio fuschii, Solidago virga-aurea) et se trouve tapissé, en hiver et au printemps, par un lit de feuilles tassées par la neige. Les fonds de doline sont caractérisés par une absence de régénération naturelle et de végétation arbustive. Ce ne sont cependant pas des stations impropres à l'état boisé comme en témoigne le grand développement des sapins implantés dans ces sites. Les stations de "replat" s'étendent en bordure des dépressions karstiques. Elles sont recouvertes d'une végétation arbustive abritant une strate muscinale (Rhytidiadelphus loreus), et sont caractérisées par un haut niveau de régénération. Dans les 2 stations, les sols sont brunifiés, lessivés, profonds et normalement drainés. Les matériaux minéraux, qui proviennent du résidu insoluble des calcaires locaux et d'apport de poussières éoliennes (Pochon, 1978; Gaiffe et Bruckert, 1985), sont limono-argileux. L'horizon argilique et la position géomorphologique qui les caractérisent, les classent parmi les sols bruns lessivés colluviaux (CPCS, 1967). Les analyses de 2 pédons sont données dans le tableau I. Les hori-

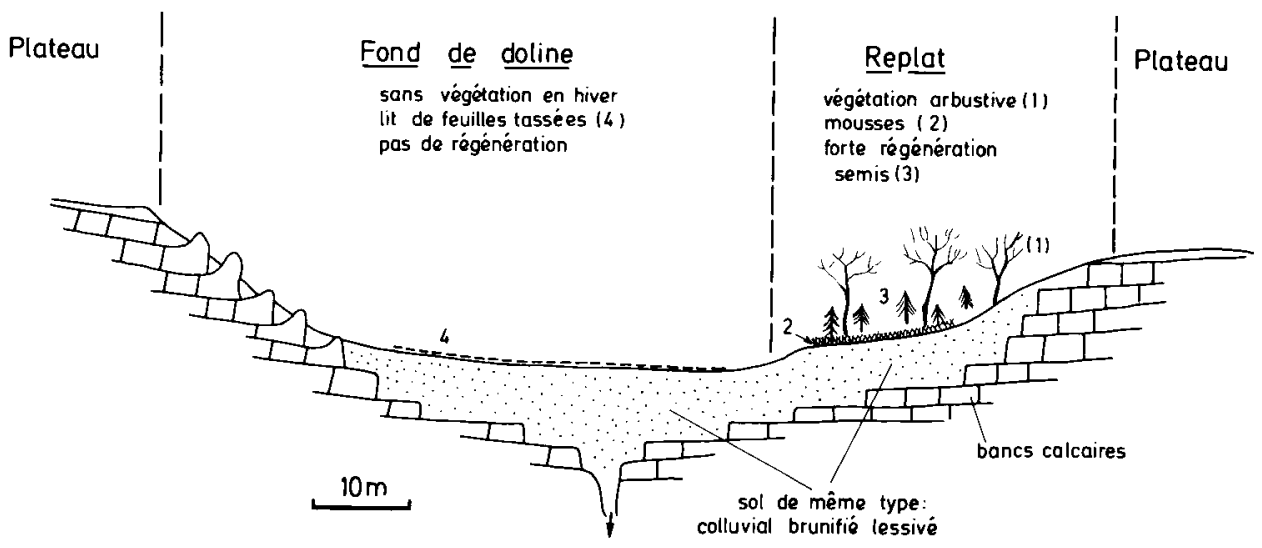

Fig 1. Caractéristiques des deux stations étudiées : fond de doline et replat. 


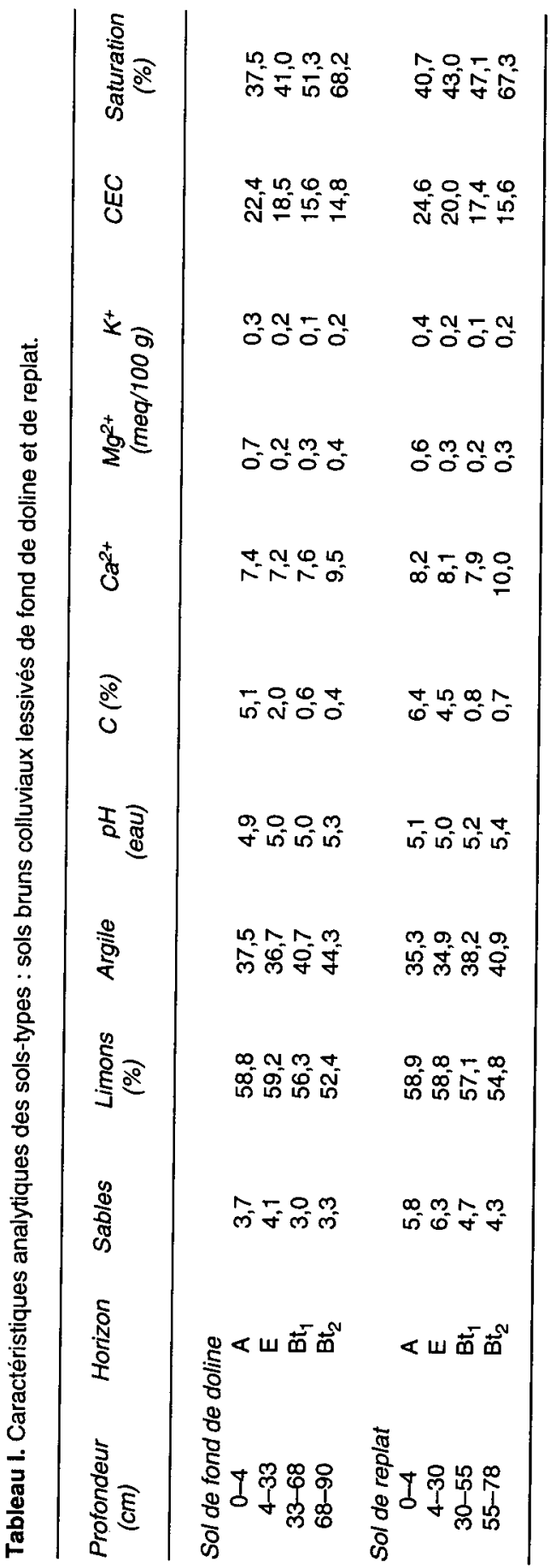


zons $A$ et $E$ bruns jaunâtres ( 10 YR $7 / 8$ et $6 / 8$ ), sont limoneux, en agrégats fins, très poreux avec de nombreuses galeries, très prospectés par des racines; les horizons $\mathrm{Bt}_{1-2}$ bruns $(7,5$ YR 5/6 et 5 YR 5/8), sont argilo-limoneux, polyédriques avec des argilanes.

\section{Expérimentation in situ}

Trois stations de fond de doline et trois stations de replat ont été équipées d'un thermohygrographe pour mesurer la température et l'humidité de l'air, sous abri, à $2 \mathrm{~cm}$ au-dessus du sol, d'une thermosonde pour mesurer la température des sols à $-10 \mathrm{~cm}$ de profondeur, de tensiomètres pour suivre le potentiel hydrique à -10 $\mathrm{cm}$ (3 répétitions par station), d'un pluviographe installé dans une clairière proche pour déterminer les précipitations. Les stations étudiées ont des dimensions suffisamment grandes pour laisser les sites expérimentaux hors de l'ombrage des peuplements.

Dans chaque station, des lots de 70 graines sélectionnées ont été semés en automne sur deux carrés permanents de $35 \times 35 \mathrm{~cm}$ par station. Les graines, qui provenaient de la forêt de la Joux, avaient un poids moyen de $4010^{-3} \mathrm{~g}$ (lot $\mathrm{HI}-82-400$ de porte-graine d'âge moyen de 90 ans). Elles avaient été triées et débarrassées de leur aile par traitement mécanique effectué à la sècherie de Joux.

Une fois semées, les graines ont été protègées contre l'attaque des rongeurs par des cages grillagées (Godan, 1983). Durant tout I'hiver, les graines ont été recouvertes par la neige. Au printemps, après la germination, les cages grillagées ont été enlevées sur la moitié des carrés permanents. $\dot{A}$ partir des premiers jours de mai, nous avons effectué 2 observations par semaine et visité les sites à l'occasion de chaque événement climatique : fonte des neiges, pluies orageuses. À chacune de nos visites, nous avons noté : le nombre de semis enracinés; le nombre de semis déplacés; le nombre de semis déracinés et abîmés; le nombre de semis disparus; le nombre de semis détériorés par les limaces ou d'autres phytophages (carrés sans cages de protection).

Les expériences ont été répétées pendant 3 ans, de 1983 à 1986.

\section{Expérimentations en conditions contrôlées}

Les expérimentations ont été conduites en serre à température constante de $20^{\circ} \mathrm{C}$ avec un éclairement correspondant à $20 \mathrm{~W} \cdot \mathrm{m}^{-2}$ d'énergie incidente dans la lumière visible pendant $12 \mathrm{~h}$, fournie par un éclairage naturel indirect. Des graines de même poids et de même origine que dans l'expérimentation in situ, après avoir été placées à $4^{\circ} \mathrm{C}$ pendant 4 semaines pour lever leur dormance, ont été mises à germer sur un terreau sableux dont la composition est donnée au tableau II. Quand les radicules ont atteint 3 $\mathrm{mm}$, les graines ont été partagées en 2 lots pour effectuer les expériences suivantes :

\section{Expérience 1}

Les graines germées ont été disposées horizontalement à la surface du terreau remplissant 4 boites en plastique de $15 \times 9,5 \mathrm{~cm}$ et de $5,5 \mathrm{~cm}$ de haut. Chaque boîte a reçu 8 graines germées. Le fond est percé de trous pour assurer un drainage libre. Le terreau est arrosé de façon à le ramener à la capacité au champ tous les 7 jours; entre 2 arrosages, l'humidité s'est abaissée au plus à $50 \%$ de la réserve utile en eau. Deux boîtes ont été recouvertes par une cloche de façon à maintenir un environnement atmos-

Tableau II. Caractéristiques chimiques et physico-chimiques du terreau utilisé.

\begin{tabular}{lrll}
\hline Sables & $92,7 \%$ & $\mathrm{Ca}^{2+}$ & \\
Limons & $3,9 \%$ & $\mathrm{Mg}^{2+}$ & $6,90 \mathrm{~g} / \mathrm{kg}$ \\
Argile & $3,8 \%$ & $\mathrm{~K}^{+}$ & $0,11 \mathrm{~g} / \mathrm{kg}$ \\
C organique & $3,7 \%$ & $\mathrm{CEC}$ & $0,04 \mathrm{~g} / \mathrm{kg}$ \\
$\mathrm{N}$ total & $0,2 \%$ & $\mathrm{pH}($ eau $)$ & $5,60 \mathrm{meq} / 100 \mathrm{~g}$ \\
& & 7,5 \\
\hline
\end{tabular}


phérique humide (traitement air saturé). Deux boîtes ont été placées à une humidité relative de 30 à $40 \%$ (traitement air sec).

L'expérience a duré 21 jours au cours desquels, nous avons : 1) observé le mode de développement de la radicule et de l'hypocotyle; 2) déterminé le taux de survie des semis.

\section{Expérience 2}

Les graines germées ont été plantées verticalement jusqu'au tiers de leur longueur dans le terreau remplissant 60 pots de terre cuite de 10 $\mathrm{cm}$ de diamètre sur $9 \mathrm{~cm}$ de haut. Chaque pot a reçu une graine germée.

L'humidité du terreau a été amenée à la capacité au champ et maintenue à cette valeur pendant 5 jours afin de permettre à la radicule d'entamer sa phase de croissance rapide. À partir du $5^{\ominus}$ jour et jusqu'au $53^{\ominus}$ jour, l'humidité des substrats et l'hygroscopicité de l'air ont été réglées de façon à obtenir les traitements suivants : 1) substrat à l'humidité équivalente et air saturé (10 répétitions); 2) substrat à l'humidité équivalente et air sec (20 répétitions); 3) substrat avec déficit hydrique et air saturé (10 répétitions); 4) substrat avec déficit hydrique et air $\sec$ (20 répétitions).

Dans les traitements 1 et 2 , le terreau a été ramené à la capacité au champ tous les 7 jours; entre 2 arrosages, l'humidité s'est abaissée au plus à $50 \%$ de la réserve utile. Dans les traitements 3 et 4 , le terreau n'a été ramené à la capacité au champ que tous les 14 jours; entre 2 arrosages, l'humidité s'est abaissée jusqu'à une valeur proche du point de flétrissement.

À 53 jours, 10 plantules par traitement ont été débarrassées du terreau par lavage, dans le but de mesurer la longueur de la radicule. Avec les plantules restantes pour les traitements 2 et 4, l'expérience a été poursuivie jusqu'à 365 jours, ie au stade "système racinaire développé". Les radicules étant bien implantées, l'alimentation en eau des semis a été assurée par l'intermédiaire de l'humidité des substrats (air non saturé). Les traitements ont donc été ramenés aux 2 protocoles suivants : substrat à l'humidité équivalente (10 plantules); substrat avec déficit hydrique (10 plantules).

À 365 jours, 5 plantules par traitement (ie la moitié des sapins) ont été débarrassées du terreau par lavage, dans le but de mesurer la lon- gueur des racines principales et secondaires. Avec les 5 plantules restantes par traitement, l'expérience suivante a été réalisée : les pots ont été arrosés; puis le terreau ayant été ressuyé pendant 3 jours, les plantules ont été exposées au soleil pendant $2 \mathrm{~h}\left(650 \mathrm{~W} \cdot \mathrm{m}^{-2}\right)$, à la suite de cet ensoleillement, les plantules ont été placées en observation pour suivre leur comportement (retour à $20 \mathrm{~W} \cdot \mathrm{m}^{-2}$ ).

\section{RÉSULTATS}

\section{Données climatiques in situ}

Les données microclimatiques et pédoclimatiques observées de mai à septembre 1986 sont présentées dans la figure 2. Les précipitations ont été : 1) abondantes en mai et pendant les 2 premières décades de juin (phase humide avec $115 \mathrm{~mm}$ ); 2) intermittentes avec des périodes sèches du 20 juin au 10 juillet; 3 ) rares du 10 juillet jusqu'à la deuxième décade d'août (phase sèche); 4) de nouveau abondantes en fin août et septembre (phase humide). Les températures se sont progressivement élevées de mai à juillet, puis se sont maintenues à ce niveau pendant les mois d'août et septembre (voir la température des sols).

Pendant la première phase humide, les sols des deux stations ont conservé un potentiel matriciel total bas, correspondant à celui de la capacité au champ : le pédoclimat est apparu semblable dans les deux sols. Par contre, I'humidité relative et la température de l'air, mesurées à $2 \mathrm{~cm}$ du sol sous abri, ont opposé les deux stations (moyennes statistiquement différentes à la probabilité de 0,05$)$. Les fonds de doline, qui étaient sans végétation à cette époque, ont été à la fois plus secs (comparer les données minimales) et plus chauds (données maximales) que les replats. Ces derniers étant couverts de mousses, les mesures ont été prises au sein des mousses. 
Facteurs physiques et régénération du sapin

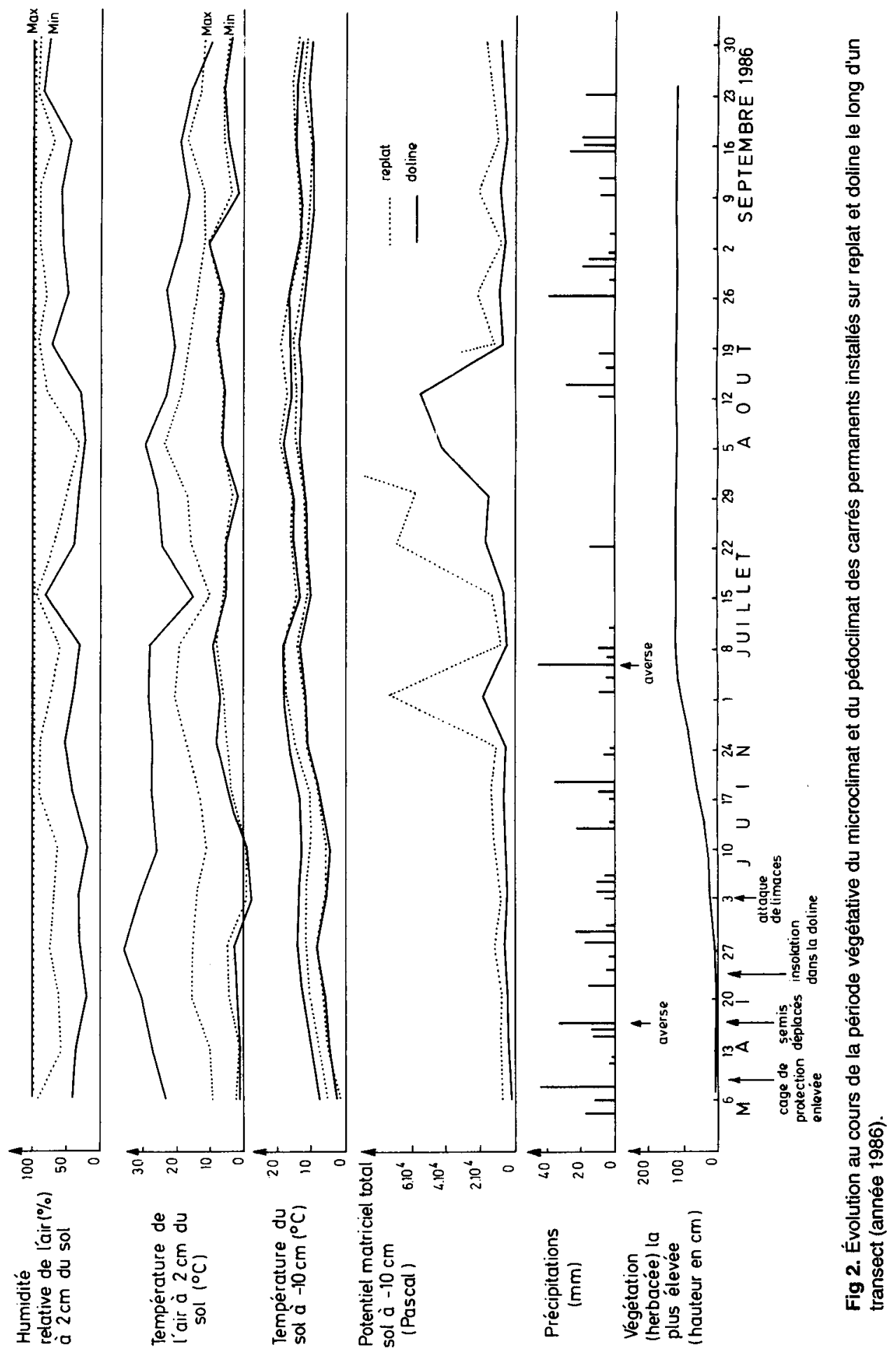


Pendant la deuxième phase (sèche), les sols ont réagi rapidement à l'absence de pluies, les sols des replats s'étant désséchés plus fortement que ceux des fonds de doline. Les alternances d'humectationdessiccation ont eu des effets plus marqués dans les premiers que dans les seconds (moyennes différentes au seuil de $0,05)$. Pendant cette phase, l'humidité relative et la température de l'air ont continué d'opposer les 2 stations, mais de façon plus atténuée parce que la végétation s'est développée dans les deux stations : strate herbacée dans les dolines, feuillage des arbustes dans les replats.

Pendant la troisième phase (humide), les sols sont revenus à la capacité au champ, alors que l'humidité relative et la température de l'air ont eu tendance à s'égaliser dans les 2 stations.

\section{Comportement des semis in situ}

La germination des graines, protégées de l'attaque des prédateurs, s'est déclenchée au cours des 3 premières semaines du mois de mai, à la fin de la fonte des neiges. Elle s'est échelonnée sur 8 jours. Les taux de germination des graines analysées pendant 3 ans in situ, ont été de l'ordre de $30 \%$ dans l'ensemble des stations (tableau III).

Les germes se sont développés d'abord à la surface du sol, sans géotropisme pendant plusieurs jours (au moins 5 jours) : les radicules ont rampé sur le substrat sans s'y implanter. Les radicules se sont ensuite recourbées pour s'orienter vers le sol (morphologie en crosse).

Une semaine après la germination, on a noté que la plupart des semis ont été déplacés. Le phénomène a été provoqué par les averses violentes qui surviennent régulièrement au printemps dans le Jura, comme en ont témoigné des averses dé- passant $20 \mathrm{~mm}^{\bullet \mathrm{h}^{-1}}$, enregistrées par les pluviographes 3 années de suite. Cependant, les taux de déplacement (en \% des graines germées) ont beaucoup différé selon les stations : ils ont atteint $98 \%$ dans les fonds de doline contre $21 \%$ seulement dans les replats (tableau IV).

Au bout du premier mois qui a qui suivi la germination, les plantules étant protégées de l'attaque des prédateurs, le taux de survie des semis (en \% des graines germées) a opposé les deux stations : $3 \%$ dans les fond de doline contre $91 \%$ dans les replats (tableau $\mathrm{V}$ ). Toujours au bout

Tableau III. Comportement des semis in situ : taux de germination (moyennes de 6 mesures, l'écart type ne dépassant pas 3). Les mêmes lettres signifient que les moyennes ne sont pas statistiquement différentes à la probabilité de 0,01 (test $t$ de Student).

Taux de germination

(\%)

\begin{tabular}{llll}
\cline { 2 - 4 } Stations & \multicolumn{3}{c}{$(\%)$} \\
& 1984 & 1985 & 1986 \\
& & & \\
Fonds de doline & $31 \mathrm{a}$ & $34 \mathrm{a}$ & $32 \mathrm{a}$ \\
Replats & $33 \mathrm{a}$ & $30 \mathrm{a}$ & $31 \mathrm{a}$ \\
\hline
\end{tabular}

Tableau IV. Comportement des semis in situ : taux de déplacement des semis en $\%$ des graines germées (moyennes de 6 mesures, l'écart type ne dépassant pas 4). Les mêmes lettres dans les colonnes signifient que les moyennes ne sont pas statistiquement différentes à la probabilité de 0,01 (test $t$ de Student).

\begin{tabular}{|c|c|c|c|c|}
\hline \multirow[t]{2}{*}{ Stations } & \multicolumn{3}{|c|}{$\begin{array}{c}\text { Taux de déplacement } \\
(\%)\end{array}$} & \multirow[t]{2}{*}{$\begin{array}{l}\text { Moyenne } \\
\text { sur } 3 \text { ans }\end{array}$} \\
\hline & 1984 & 1985 & 1986 & \\
\hline $\begin{array}{l}\text { Fond de doline } \\
\text { Replats }\end{array}$ & $\begin{array}{r}100 a \\
18 b\end{array}$ & $\begin{array}{r}100 a \\
33 b\end{array}$ & $\begin{array}{l}95 a \\
13 b\end{array}$ & $\begin{array}{l}98 a \\
21 b\end{array}$ \\
\hline
\end{tabular}


Tableau V. Comportement des semis in situ : taux de survie en \% des graines germées, protégées ou non contre les prédateurs (moyennes de 3 mesures). Les mêmes lettres signifient que les moyennes ne sont pas statistiquement différentes à la probabilité de 0,01 (test $t$ de Student).

\begin{tabular}{lrrrrr}
\hline Stations & 1984 & 1985 & 1986 & Moyenne sur 3ans & Écart type \\
\hline Avec cages de protection & & & & & \\
$\begin{array}{l}\text { Fonds de doline } \\
\text { Replats }\end{array}$ & $3^{\mathrm{a}}$ & $4^{\mathrm{a}}$ & $2^{\mathrm{a}}$ & $3^{\mathrm{a}}$ & 1 \\
Sans cages de protection & $97^{\mathrm{b}}$ & $91^{\mathrm{b}}$ & $83^{\mathrm{b}}$ & $91^{\mathrm{b}}$ & 6 \\
$\begin{array}{l}\text { Fonds de doline } \\
\text { Replats }\end{array}$ & $0^{\mathrm{c}}$ & $0^{\mathrm{c}}$ & $0^{\mathrm{c}}$ & $0^{\mathrm{c}}$ & \\
& $38^{\mathrm{d}}$ & $28^{\mathrm{d}}$ & $22^{\mathrm{d}}$ & $29^{\mathrm{d}}$ & 7 \\
\hline
\end{tabular}

du premier mois, mais sans protection contre les prédateurs, le taux de survie a différencié aussi des deux stations, avec cependant beaucoup moins d'écart : respectivement 0 et $29 \%$.

Au bout d'un an, les taux de survie se sont encore abaissés dans les replats, de 91 à $84 \%$ (plantules protégées) et de 29 à $26 \%$ (plantules non protégées de l'attaque des prédateurs).

\section{Comportement des semis et des plantules in vitro}

Dans l'expérience 1 (graines germées disposées horizontalement à la surface du substrat, comme le milieu naturel), I'hypocotyle portant à son extrémité les feuilles cotylédonaires s'est dressé verticalement au-dessus du substrat et la radicule a décrit une sinusoïde avant de pénétrer et de s'enfoncer verticalement dans le substrat (fig 3). Dans tous les essais, la première phase de croissance racinaire s'est effectuée parallèlement au substrat ou même parfois en sens opposé (courbure vers le haut). La radicule a d'abord rampé à la surface du substrat avant de s'orienter vers lui. Dans un environnement humide (traitement air saturé) les plantules se sont bien développées et le taux de survie au $21^{\mathrm{e}}$ jour a atteint $93,7 \%$. À une humidité relative de 30 à $40 \%$ (traitement sec), la plupart des plantules se sont désséchées et le taux de survie est tombé à $6,3 \%$.

Dans l'expérience 2 (graines germées plantées dans le substrat jusqu'au tiers de leur longueur), les radicules se sont enfoncées d'emblée verticalement dans le substrat (fig 3). Les semis plantés sur substrat à l'humidité équivalente ou sur substrat avec déficit hydrique mais en environnement atmosphérique humide se sont démarqués par un développement élevé des radicules (moyennes [1] : 69,3 et $69,5 \mathrm{~mm}$ à 53 jours). Les semis plantés sur substrat humide et placés en air sec se sont caractérisés par une radicule un peu moins longue (moyenne [2] : 64,1 $\mathrm{mm})$, mais la différence avec les moyennes précédentes n'a pas été significative (test $t$ de Student). Les semis plantés sur substrat avec déficité hydrique et à une humidité relative de $40 \%$ (air sec), ont présenté par contre des radicules moins longues (moyenne [3] : $54,3 \mathrm{~mm}$, différence significative au seuil 0,01 avec (1) et 0,05 avec (2). 

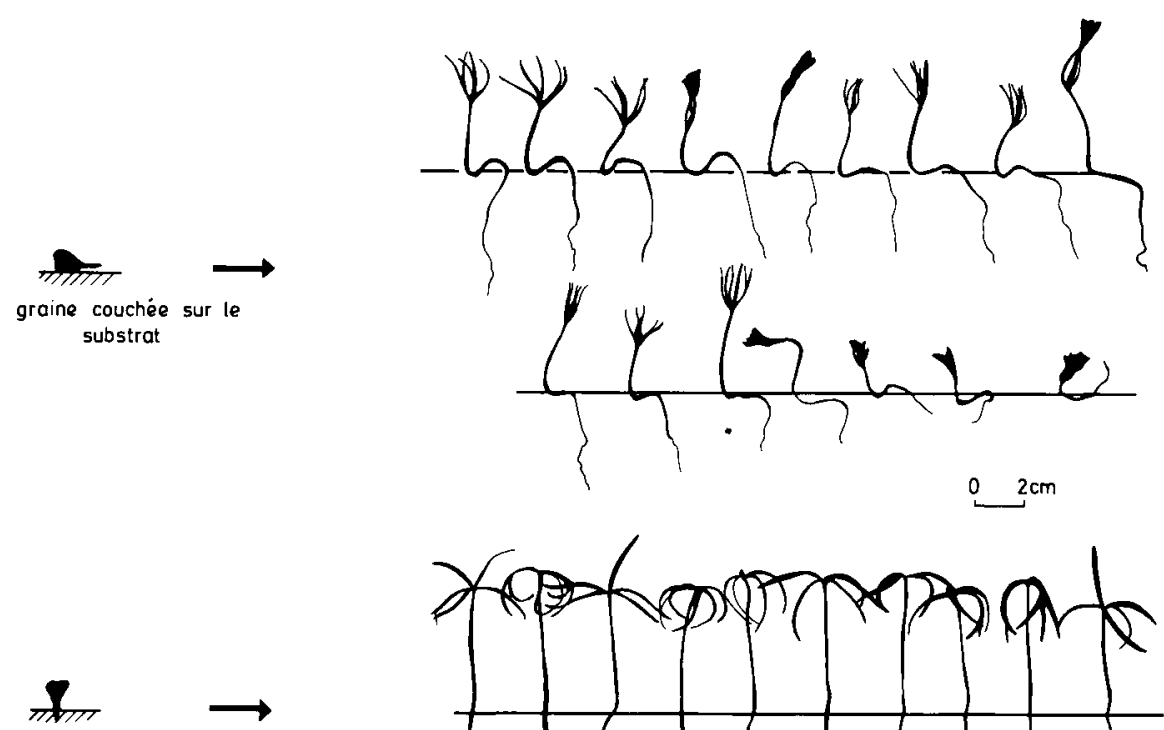

graine plantée verticalement dans lo substrat

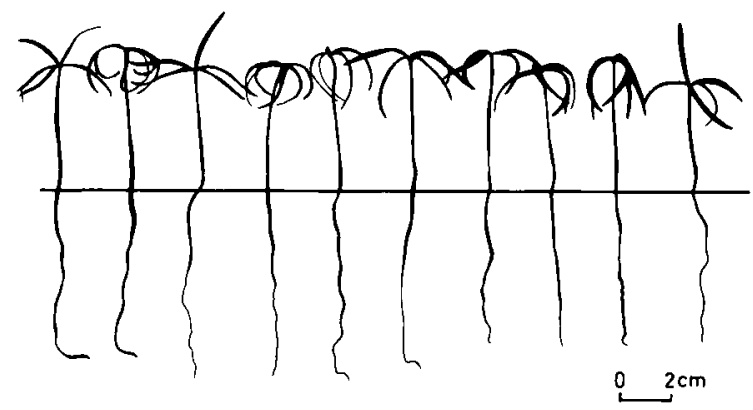

Fig 3. Comportement des semis en fonction de la position initiale des graines sur le substrat.

Au cours de l'année qui a suivi, l'hypocotyle et le bourgeon terminal n'ont pas changé de taille. Les systèmes racinaires (somme des longueurs des racines principales et secondaires) se sont développés, mais ont atteint des dimensions très différentes selon les traitements : sur substrat à l'humidité équivalente le système racinaire a atteint 357,6 et $116,3 \mathrm{~mm}$ sur substrat soumis à des phases sèches (moyennes significativement différentes au seuil de 0,01). Les taux de survie des plantules ont été de $100 \%$ sur substrat frais et de $90 \%$ sur substrat avec déficit hydrique.

Les plantules qui ont été soumises à un ensoleillement direct de $2 \mathrm{~h}$ se sont comportées très différemment selon les traitements antérieurs. Celles qui possédaient un système racinaire bien développé (traitements à l'humidité équivalente) sont toutes restées turgescentes (taux de survie à $24 \mathrm{~h}$ $=100 \%$ ); par contre, celles qui avaient un système racinaire réduit sont flétries rapidement : les taux de survie sont tombés à $50 \%$ au bout de $12 \mathrm{~h}$ et $0 \%$ au bout de $24 \mathrm{~h}$.

\section{DISCUSSION ET CONCLUSION}

Au vu du comportement des semis d'une part et des données microclimatiques et pédoclimatiques disponibles, il ressort que le mois qui suit la germination, de fin mai à fin juin, constitue une première phase critique, qui est déterminante pour la survie des semis placés dans les fonds de doline. 
Une deuxième phase critique, de fin juin à fin août, peut aussi devenir déterminante dans les replats pour les plantules mal enracinées à cette époque.

Les averses orageuses au printemps constituent l'un des traits les moins connus et pourtant les plus spectaculaires du climat jurassien. En cette période de l'année où les sols ne sont pas protégés par la végétation herbacée encore inexistante, la violence des averses provoque des transports de matière qui touchent non seulement les sols (Gaiffe et Bruckert, 1985; Gaiffe, 1990) mais également les matériaux peu ou non fixés au substrat, en particulier les graines germées, les semis rampants et même les semis implantés (Tan, 1987).

Les fonds de doline, recouverts de feuilles tassées par la neige, offrent une surface difficilement pénétrable sur laquelle les semis sont soumis à une action très dommageable des pluies battantes. Dans les replats, revêtus d'un tapis muscinal et d'arbustes, les semis sont protégés de l'effet des averses.

Le déplacement des semis, déjà signalé chez le pin Weymouth (Pinus strobus) aux États-Unis (Cléments, 1964), dépend à la fois de la violence des précipitations et de la «rugosité» du terrain. L'énergie des précipitations est fortement atténuée par les couvertures muscinales (Lieutaghi, 1972).

Le déplacement des semis entraîne des lésions au niveau de la radicule. L'insolation pendant les périodes de beau temps qui alternent avec les averses en cette saison, provoque la mortalité, par dessiccation, des semis déplacés. Les dégâts observés apparaissent beaucoup plus importants dans les fonds de doline que dans les replats. Le dessèchement de semis peu ou non enracinés a été décrit également chez Pinus strobus (Smith, 1951). L'importance des replats vis-à-vis de l'implantation des forêts a été signalée au $\mathrm{Ca}$ - nada par Brown (1981) et dans les Alpes par Mullenbach (1982).

L'humidité de l'air joue un rôle fondamental dans les premiers stades de développement des plantules et semble être une condition nécessaire pour assurer leur survie pendant la phase initiale de géotropisme nul. L'alimentation en eau par l'air saturé constitue encore un facteur déterminant favorable au développement des plantules lorsque des radicules se trouvent implantées en substrat sec. Cette observation implique que les plantules sont en partie alimentées en eau par l'intermédiaire des tissus aériens, comme cela a été constaté chez d'autres plantes (Vartanian, 1977).

Au total, les paramètres microclimatiques et pédoclimatiques suivants opposent les 2 stations étudiées : l'humidité relative et la température de l'air, l'ensoleillement, les régimes hydriques internes des sols. Les facteurs microclimatiques peuvent exercer une action déterminante pendant la première phase humide, entre fin mai et fin juin, période pendant laquelle l'ensoleillement peut atteindre directement les fonds de doline dépourvus de couverture végétale à cette époque. Les facteurs pédoclimatiques prennent ensuite le relais pendant la période de fin juin à fin août.

Les facteurs microclimatiques et pédologiques agissent donc sur les premiers stades de développement et d'implantation des semis de sapin. Ce constat n'interdit pas que d'autres facteurs puissent intervenir pendant cette phase précoce de la régénération naturelle et par la suite : gelées tardives (Tranquillini, 1979), si fréquentes et actives dans le Jura (Tan, 1983); facteurs biotiques tels les prédateurs (Wojnar, 1970; Tan, 1987) et diverses substances toxiques minérales ou organiques (Rousseau, 1960; Becker et Drapier, 1984 et 1985). 
Les conclusions relatives à l'importance des facteurs physiques mis en évidence en forêt de Levier semblent pouvoir s'appliquer à l'ensemble du Jura et à des massifs de même altitude, géologiquement semblables, de l'arc alpin, parce que les modèles géomorphologiques et stationnels concernés sont répétitifs dans les contextes karstiques de ces régions.

On peut enfin penser que certains des facteurs physiques étudiés pourraient aussi être importants hors du contexte régional. Les rôles de piège et de protection joués par les mousses ont été pressentis par Rousseau (1960), Brossier (1977) et Drapier (1983) dans les Vosges et les Alpes, mais sans que les mécanismes en cause aient été étudiés.

\section{RÉFÉRENCES}

Aubert D (1969) Phénomènes et formes du karst jurassien. Eclogae Geol Helv 62 (2), 325-399

Badre L (1962 La sapinière vosgienne et le gibier. Bull Soc For Franche-Comté et des Provinces de l'Est 1, 473-480

Becker M, Bennett P (1980) Propriétés allèlopathiques d'une graminée forestière : la grande fétuque (Festuca silvatica Vill) CR $6^{\circ}$ Coll Intern Ecol Biol Système Mouv Herbes, Columa-EWRS, Montpellier, tome 2, 451-460

Becker M, Drapier J (1984) Rôle de l'allélopathie dans les difficultés de régénération du sapin (Abies alba Mill). I. Propriétés phytotoxiques des hydrosolubles d'aiguilles de sapin. Acta CEcol CEcol Plant 5 (19), 347-356

Becker M, Drapier J (1985) Rôle de l'allélopathie dans les difficultés de régénération du sapin (Abies alba Mill). II. Étude des lessivats naturels de feuillage, de litière et d'humus. Acta $C E c o l C E c o l$ Plant 6 (20), 31-40

Brinar M (1971) The effect of "Kolins" on seed germination in connection with the alternation of certain forest tree species. Godz Westn 29 (2/3), 65-83

Brossier J (1977) Évolution des idées en matière forestière et conséquences sur la ges- tion des forêts alpines. Rev For Fr spéc, 153161

Brown JL (1981) Les forêts du Témiscamingue, Québec. Écologie et photo-interprétation. Études écologiques. Lab écol For, Université Laval, Québec, 245-258

Bruckert S, Gaiffe M (1980) Analyse des facteurs de formation et de distribution des sols en pays calcaire glaciaire ou karstique. Plaine de Frasne - Bonnevaux et Montagne du Laveron - Jura Central. Ann Sci Univ Franche-Comté Besançon Biol Vég sér 4, 1, 19-65

Bruckert S, Tan BS (1986) Ėtude du dépérissement des forêts de l'étage montagnard du Jura en relation avec les sols calciques ou acides du Karst, l'importance de leurs éléments nutritifs et l'état nutritionnel des peuplements. Ann Sci Univ Franche-Comté Besançon, Biol Vét Sér 4, 6, 37-44

Cléments JR (1984) The potential influence of soil splashing by raindrops on seedling survival. For Chron 40 (4), 512-513

CPSC (1967) Classification française des sols. Doc offset, INRA, $96 \mathrm{p}$

Drapier J (1983) Les difficultés de régénération des sapinières vosgiennes. Importance de I'humus et rôle de l'allélopathie. Thèse, Univ Nancy I, STMCM, 109 p

Ferrari GA, Wolf U (1970) Considerazioni sui soli e la rinnovazione naturale dell' Abete bianco (Abies alba Mill) del bosco "Abieti soprani" (Pescopennataro - is). Ann Accad Ital Sci For 19, 423-439

Gabellini B, Screm E (1968) The suitability of various natural substrates to serve as a seedbed to Abies alba and Picea abies. Ann Accad Ital Sci For 17, 400-409

Gaiffe M (1990) Erosion of calcimagnesic soils as aggregates in Jurassian pedological systems. Catena 17 (2), 141-149

Gaiffe M, Bruckert S (1985) Analyse des transports de matières et des processus pédogénétiques impliqués dans les chaînes de sols du karst jurassien. Catena Soils and Geomorphol suppl 6, 159-174

Gaiffe M, Bruckert S (1991) Déterminisme paléoécologique des écosystèmes actuels du Haut-Jura, en relation avec la fracturation des roches. Ann Sci For 48, 575-591

Godan D (1983) Pest slugs and snails. SpringerVerlag, Berlin, $26 \mathrm{p}$ 
Lieutaghi P (1972) L'environnement végétal. Delachaux et Niestlé, Neuchatel, 60-61

Mullenbach $P(1982)$ Les reboisements au voisinage de la limite altitudinale de la végétation forestière (limite sylvestre). Exemple de la station du Chazelet. Premiers résultats. Rev For Fr 5, spécial

Nicod J (1990) Le karst jurassien. Modèle géomorphologique spécifique. Bull Soc Neuch Sci Nat 113, 13-25

Pochon M (1978) Origine et évolution des sols du Haut-Jura suisse. Phénomènes d'altération des roches calcaires sous climat tempéré humide. Thèse, Univ Neuchâtel, $192 p$

Roques A (1988) La spécificité des relations entre cônes de cônifères et insectes inféodés en Europe Occidentale : un exemple d'étude des interactions plantes-insectes. Thèse, Univ Pau, $225 \mathrm{p}$

Rousseau $L Z$ (1960) De l'influence de type d'humus sur le développement des plantules de sapin dans les Vosges. Ann Ec Nat Eaux et For, 17, 17-115

Smith DM (1951) The influence of seedbed conditions on the regeneration of Eastern White pine. Conn Agric Bull 545, 7-61
Tan BS (1983) Rôles du sol dans la régénération du sapin et du climat dans le reboisement des combes à l'étage montagnard du Jura. Thèse, Univ Nancy I, $131 p$

Tan BS (1987) Étude de l'influence de quelques facteurs écologiques sur les premiers stades de la régénération du sapin (Abies alba) à l'étage montagnard du Jura. Rev Ecol Biol Sol 24 (4), 623-635

Tan BS (1988) La régénération du Sapin (Abies alba Mill) dans le Jura : influence des facteurs physiques, édaphiques et biotiques sur les stades précoces du développement des semis. Thèse, Univ Besançon, $133 p$

Tranquillini W (1979) Physical ecology of the alpine timberline. Springer-Verlag, Berlin

Vallée G (1967) Nouvelles contributions à l'étude du rôle du manganèse dans la régénération de la sapinière vosgienne. Thèse, Univ Nancy I, $138 p$

Vartanian N (1977) Influence des facteurs hydriques de l'environnement sur le système racinaire : aspects morphologiques histologiques et écophysiologiques. Thèse, Univ Orsay, $150 \mathrm{p}$

Wojnar T (1970) Results of experimental sowings of Abies alba in the forest of Marescu. Ann Accad Ital Sci For 19, 217-241 